\title{
Second-order correlation function of a phase fluctuating Bose-Einstein condensate
}

\author{
L. Cacciapuoti,* D. Hellweg, M. Kottke, T. Schulte, W. Ertmer, and J. J. Arlt \\ Institut für Quantenoptik, Universität Hannover, Welfengarten 1, 30167 Hannover, Germany \\ K. Sengstock \\ Institut für Laserphysik, Universität Hamburg, Luruper Chaussee 149, 22761 Hamburg, Germany \\ L. Santos and M. Lewenstein \\ Institut für Theoretische Physik, Universität Hannover, Appelstraße 2, 30167 Hannover, Germany
}

(Received 8 July 2003; published 20 November 2003)

\begin{abstract}
The coherence properties of phase fluctuating Bose-Einstein condensates are studied both theoretically and experimentally. We derive a general expression for the $\mathrm{N}$-particle correlation function of a condensed Bose gas in a highly elongated trapping potential. The second-order correlation function is analyzed in detail, and an interferometric method to directly measure it is discussed and experimentally implemented. Using a Bragg diffraction interferometer, we measure intensity correlations in the interference pattern generated by two spatially displaced copies of a parent condensate. Our experiment demonstrates how to characterize the secondorder correlation function of a highly elongated condensate and to measure its phase coherence length.
\end{abstract}

DOI: $10.1103 /$ PhysRevA.68.053612

PACS number(s): 03.75.Hh, 03.75.Nt, 39.20.+q

\section{INTRODUCTION}

Among the various topics related to the exciting field of Bose-Einstein condensation (BEC) [1], the analysis of coherence properties of degenerate Bose gases has attracted major interest. Coherence plays a key role in the understanding of the fundamentals of BEC and has a crucial importance for many promising BEC applications, such as matter wave interferometry, guided atomic beams, and atom lasers. The coherent character of trapped three-dimensional (3D) condensates well below the BEC transition temperature $T_{c}$ has been confirmed by several experiments, using interferometric $[2,3]$ and spectroscopic methods [4].

However, recent theoretical and experimental developments have shown that phase coherence is far from being an obvious property of BEC. In particular, a phase fluctuating BEC at equilibrium has been theoretically predicted in onedimensional [5], two-dimensional [6,7], and even in highly elongated, but still three-dimensional [8] trapped Bose gases. Interestingly, in these cases the density distribution does not differ from the usual BEC profile, since density fluctuations are largely suppressed by the repulsive mean-field potential. These systems are commonly called quasicondensates. Phase fluctuations can be induced either by quantum [9] or by thermal fluctuations [10]. For typical experimental temperatures quantum phase fluctuations can safely be neglected as long as the system remains in the weakly interacting regime [11]. The amplitude of phase fluctuations, therefore, depends strongly on temperature and trapping geometry. In this sense, a nearly phase coherent BEC in a highly elongated trap can be achieved only far below $T_{c}$, imposing severe limitations on experiments in constrained geometries. Phase-fluctuating

\footnotetext{
*Present address: BNM-SYRTE, Observatoire de Paris, 61 Avenue de l'Observatoire, 75014 Paris, France. Email address: Luigi.Cacciapuoti@obspm.fr
}

BECs have been the subject of recent theoretical efforts, including the development of a modified mean-field theory valid in all dimensions and all temperatures below the critical point $[12,13]$, the analysis of dynamic correlation functions [14], and the extension of Bogoliubov theory to lowdimensional degenerate Bose gases [15].

The phase-fluctuating nature of highly elongated BECs was first experimentally demonstrated in Ref. [16]. During the ballistic expansion, phase fluctuations transform into density modulations. The appearance of phase fluctuations and their statistic nature were studied and the dependence of their average value on experimental parameters was characterized $[16,17]$. Moreover, the results obtained from measurements of the energy released during the expansion confirmed the absence of density fluctuations in the trapped cloud $[18,19]$. Recently, the physics of quasicondensates has been studied by means of Bragg spectroscopy, showing that the existence of phase fluctuations leads to an observable broadening of the momentum distribution $[19,20]$. A further experiment has analyzed the phase coherence length of nonequilibrium BECs by means of a condensate-focusing technique [21].

In this paper, we present the theoretical foundation of our studies on coherence properties of phase-fluctuating condensates. We analyze the behavior of the second-order correlation function for our experimental conditions and provide a detailed discussion of the experimental technique used in Ref. [22] to measure it. This technique is based on the analysis of density correlations in the interference pattern generated by a matter wave Bragg interferometer. The general idea is similar to the original Hanbury-Brown and Twiss experiment $[23,24]$ in which the spatial second-order correlation function of a light source is characterized by measuring the correlations of intensity fluctuations in the wave field. Our method can be used to measure the phase coherence length of the condensate.

This paper is organized as follows. In Sec. II, we briefly 
review the theory of phase fluctuating Bose-Einstein condensates in 3D elongated traps [8] and analyze the evolution of the phase pattern during the ballistic expansion. The knowledge of the free dynamics of the phase is important to closely model the BEC evolution during the measurement process. In Sec. III, we study the coherence properties of the condensate and derive a general expression for the $\mathrm{N}$-particle correlation function of highly elongated 3D BECs. In Sec. IV, the experimental technique used to measure the second-order correlation function and the phase coherence length of the condensate is reviewed in detail.

\section{PHASE FLUCTUATING CONDENSATES}

In this section, we present the phase operator of a highly elongated condensate [8] and develop an analytic description for the ballistic expansion of the fluctuating phase pattern. These results, when combined with the free evolution of density modulations presented in $[16,17]$, provide a full understanding of the order parameter dynamics during the time of flight.

\section{A. Phase operator}

In the following, we consider a cylindrically symmetric condensate in the Thomas-Fermi regime, where the repulsive mean-field interaction exceeds the radial $\left(\hbar \omega_{\rho}\right)$ and the axial $\left(\hbar \omega_{z}\right)$ trap energies. At $T=0$, the density profile has the well-known shape $n_{0}(\rho, z)=n_{0 \mathrm{~m}}\left(1-\rho^{2} / R^{2}-z^{2} / L^{2}\right)$, where $n_{0 \mathrm{~m}}=\mu / g$ denotes the maximum density of the condensate, $\mu$ is the chemical potential, $g=4 \pi \hbar^{2} a / m$ the interaction constant, $m$ the atomic mass, and $a>0$ the scattering length. Under the condition $\omega_{\rho} \gg \omega_{z}$, the radial size of the condensate, given by the Thomas-Fermi radius $R=\left(2 \mu / m \omega_{\rho}^{2}\right)^{1 / 2}$, is much smaller than the axial size, which corresponds to the Thomas-Fermi length $L=\left(2 \mu / m \omega_{z}^{2}\right)^{1 / 2}$.

Due to the repulsive mean-field energy, density fluctuations are strongly suppressed in a trapped BEC. Therefore, the field operator describing the condensate can be written in the form $\hat{\psi}(\mathbf{r})=\sqrt{n_{0}(\mathbf{r})} \exp [i \hat{\phi}(\mathbf{r})]$, where the phase operator is defined by (see, e.g., Ref. [25])

$$
\hat{\phi}(\mathbf{r})=\left[4 n_{0}(\mathbf{r})\right]^{-1 / 2} \sum_{j=1}^{\infty} f_{j}^{+}(\mathbf{r}) \hat{a}_{j}+\text { H.c. }
$$

Here $\hat{a}_{j}$ represents the annihilation operator of the quasiparticle excitation with quantum number $j$ and energy $\epsilon_{j} ; f_{j}^{+}$ $=u_{j}+v_{j}$ is the sum of the excitation wave functions $u_{j}$ and $v_{j}$, obtained from the corresponding Bogoliubov-de Gennes equations. The low-energy axial modes, which are responsible for the long wavelength axial phase fluctuations, have the energy spectrum $\epsilon_{j}=\hbar \omega_{z} \sqrt{j(j+3) / 4}$ [26]. The wave functions $f_{j}^{+}$of these quasiparticle modes have the form [8]

$$
f_{j}^{+}(\mathbf{r})=\sqrt{\frac{(j+2)(2 j+3) g n_{0}(\mathbf{r})}{4 \pi(j+1) R^{2} L \epsilon_{j}}} P_{j}^{(1,1)}\left(\frac{z}{L}\right),
$$

where $P_{j}^{(1,1)}$ are Jacobi polynomials. Equations (1) and (2) show that the phase operator depends only on the axial coordinate $z$. In Sec. III, we analyze the coherence properties of the condensate by studying the correlation functions of the operator $\hat{\psi}(\mathbf{r})$.

\section{B. Evolution of the phase fluctuating pattern}

Starting from the results presented in Refs. [16,17], we analyze the evolution of phase fluctuations during the free expansion of the degenerate Bose gas. Since the trap is highly elongated, we can assume the condensate as an infinite cylinder and use the local density approximation. The time-of-flight dynamics of the order parameter is described by the scaling law $[27,28]$

$$
\psi(\rho, z, t)=\frac{\kappa(\tilde{\rho}, z, t)}{\lambda_{\rho}(t)} e^{i\left(m \dot{\lambda} \rho_{\rho} / 2 \hbar \lambda_{\rho}\right) \rho^{2}} e^{-i \mu \tilde{t} / \hbar}
$$

where $\left(m \dot{\lambda}_{\rho} / 2 \hbar \lambda_{\rho}\right) \rho^{2}$ is the quadratic phase associated with the expansion dynamics, $\lambda_{\rho}^{2}(t)=1+\omega_{\rho}^{2} t^{2}$ is the scaling coefficient, $\tilde{t}=\int^{t} d t^{\prime} / \lambda_{\rho}\left(t^{\prime}\right)^{2}$ is the rescaled time, and $\tilde{\rho}$ $=\rho / \lambda_{\rho}(t)$ is the rescaled radial coordinate. Let $\kappa_{0}=\sqrt{n_{0}}$ be the solution of the following equation:

$$
\left[-\frac{\hbar^{2}}{2 m} \nabla_{\tilde{\rho}}^{2}+\frac{m \omega_{\rho}^{2}}{2} \widetilde{\rho}^{2}+g\left|\kappa_{0}\right|^{2}-\mu\right] \kappa_{0}=0 .
$$

If we define $\kappa=\sqrt{n} \exp (i \phi)$, with $n=n_{0}+\delta n$, and substitute the scaling law of Eq. (3) into the corresponding GrossPitaevskii equation, after linearizing in $\delta n$ and $\phi$ we obtain

$$
\begin{gathered}
\frac{\partial(\delta n)}{\partial t}=\frac{\hat{\xi} \phi}{\lambda_{\rho}^{2}(t)}-\frac{\hbar}{m} \frac{\partial^{2}}{\partial z^{2}}\left(n_{0} \phi\right), \\
\frac{\partial\left(n_{0} \phi\right)}{\partial t}=-\frac{\hat{\xi}\left(\delta n / n_{0}\right)}{4 \lambda_{\rho}^{2}(t)}+\frac{\hbar}{4 m} \frac{\partial^{2}}{\partial z^{2}}(\delta n)-\frac{g n_{0}}{\hbar \lambda_{\rho}^{2}(t)}(\delta n),
\end{gathered}
$$

where $\hat{\xi}=-(\hbar / m)\left[n_{0} \nabla_{\tilde{\rho}}^{2}+\boldsymbol{\nabla}_{\tilde{\rho}} n_{0} \boldsymbol{\nabla}_{\tilde{\rho}}\right]$. The first term on the right hand side of Eq. (6) can be neglected in the ThomasFermi regime. Following Ref. [26], we average over the radial coordinates. Let $n_{I}$ be the radially integrated unperturbed density and $\delta n_{I}$ the radially integrated density fluctuations. From Eq. (6) we obtain

$$
\begin{aligned}
\phi(\tilde{z}, \tau)= & \phi(\tilde{z}, 0)+\frac{1}{8 \lambda^{2} \zeta} \frac{\partial^{2}}{\partial \tilde{z}^{2}}\left[\int_{0}^{\tau} \frac{\delta n_{I}\left(\tilde{z}, \tau^{\prime}\right)}{n_{I}\left(\tilde{z}, \tau^{\prime}\right)} d \tau^{\prime}\right] \\
& -\frac{\zeta}{2} \int_{0}^{\tau} \frac{1}{\lambda_{\rho}^{2}\left(\tau^{\prime}\right)} \frac{\delta n_{I}\left(\tilde{z}, \tau^{\prime}\right)}{n_{I}\left(\tilde{z}, \tau^{\prime}\right)} d \tau^{\prime},
\end{aligned}
$$

with $\tau=\omega_{\rho} t, \tilde{z}=z / L, \zeta=\mu / \hbar \omega_{\rho}$, and $\lambda=\omega_{\rho} / \omega_{z}$. Equation (7) can be evaluated from the known expression [16] 


$$
\frac{\delta n_{I}(\tilde{z}, \tau)}{n_{I}(\tilde{z}, \tau)}=\sum_{j} c_{j} P_{j}^{(1,1)}(\tilde{z}) \sin \left(\frac{a_{j} \tau}{1-\tilde{z}^{2}}\right) \tau^{-b_{j}},
$$

where $b_{j}=\left(\epsilon_{j} / \hbar \omega_{\rho}\right)^{2}, a_{j}=b_{j} / \zeta$, and

$$
c_{j}=\left[\frac{(j+2)(2 j+3) g}{4 \pi R^{2} L \epsilon_{j}(j+1)}\right]^{1 / 2} \frac{\left(\alpha_{j}+\alpha_{j}^{*}\right)}{2} .
$$

$\alpha_{j}$ and $\alpha_{j}^{*}$ are random variables with a zero mean value and $\left\langle\left|\alpha_{j}\right|^{2}\right\rangle=N_{j}, N_{j}$ being the occupation of the quasiparticle mode $j$. Near the trap center, $\delta n_{I} / n_{I}$ $\simeq \sum_{j} c_{j} P_{j}^{(1,1)}(\tilde{z}) \sin \left(a_{j} \tau\right) \tau^{-b_{j}}$ and hence

$$
\begin{aligned}
\phi(\tilde{z}, \tau)= & \phi(\tilde{z}, 0)+\sum_{j} c_{j}\left\{\frac{(j+3)(j+4)}{32 \zeta \lambda^{2}} P_{j-2}^{(3,3)}(\tilde{z})\right. \\
& \times \int_{0}^{\tau} d \tau^{\prime} \sin \left(a_{j} \tau^{\prime}\right)\left(\tau^{\prime}\right)^{-b_{j}}-\frac{\zeta}{2} P_{j}^{(1,1)}(\tilde{z}) \\
& \left.\times \int_{0}^{\tau} d \tau^{\prime} \frac{\sin \left(a_{j} \tau^{\prime}\right)\left(\tau^{\prime}\right)^{-b_{j}}}{1+\tau^{\prime 2}}\right\} .
\end{aligned}
$$

For large $\lambda$ and sufficiently short times of flight, the significant contribution to the phase fluctuations is due to the modes $j$ such that $\tau \ll \lambda^{2} \zeta /[j(j+3) / 4]$ and $b_{j}=j(j+3) / 4 \lambda^{2}$ $\ll 1$. Then, using Eq. (1) for $\phi(\tilde{z}, 0)$, we obtain

$$
\phi(\tilde{z}, \tau) \simeq \sum_{j} c_{j}\left\{1-\frac{1}{2} \arctan (\tau) \frac{j(j+3)}{4 \lambda^{2}}\right\} P_{j}^{(1,1)}(\tilde{z})
$$

The second term in the brackets is the correction to the phase contribution of the $j$ th mode due to the ballistic expansion. For typical times of flight (tens of milliseconds), this correction term is very small $\left(\simeq 10^{-5}\right)$ and the phase pattern can be assumed as completely frozen. Using Eq. (10), we have verified that, for our typical experimental parameters (see Sec. IV C), the phase change due to the free evolution of the condensate is less than $\pi / 10$.

\section{CORRELATION FUNCTIONS OF A PHASE FLUCTUATING CONDENSATE}

The coherence properties of a condensate are described by the correlation functions of the field operator $\hat{\psi}$. The importance of correlation functions becomes clear if we consider that most experimental signals can be modeled by using this formalism. For example, the first- and second-order correlation functions, describing the single-particle and two-particle correlation properties of the system, are connected to the visibility of fringes in an interference experiment and to the two-body collision rate in the condensate, respectively.

As discussed in Ref. [8], the single-particle correlation function of a highly elongated degenerate Bose gas can be expressed in terms of the mean square fluctuations of the phase:

$$
\left\langle\hat{\psi}^{\dagger}\left(\mathbf{r}_{1}\right) \hat{\psi}\left(\mathbf{r}_{2}\right)\right\rangle=\sqrt{n_{0}\left(\mathbf{r}_{1}\right) n_{0}\left(\mathbf{r}_{2}\right)} \exp \left\{-\left\langle\left[\delta \hat{\phi}\left(\mathbf{r}_{1}, \mathbf{r}_{2}\right)\right]^{2}\right\rangle / 2\right\},
$$

where $\delta \hat{\phi}\left(\mathbf{r}_{1}, \mathbf{r}_{2}\right)=\hat{\phi}\left(\mathbf{r}_{1}\right)-\hat{\phi}\left(\mathbf{r}_{2}\right)$ depends directly on the phase operator $\hat{\phi}$ given in Eqs. (1). At equilibrium, the population of the $j$ th quasiparticle mode, $\left\langle\hat{a}_{j}^{\dagger} \hat{a}_{j}\right\rangle$, is a random variable with mean value $N_{j}$, given by the Bose-Einstein distribution function. The appearance of phase fluctuations is a stochastic process governed by the temperature $T$ of the system. Since individual realizations are not predictable, we average over an ensemble of identically prepared condensates in thermal equilibrium at temperature $T$. This average is indicated by $\langle\cdots\rangle_{T}$. When $k_{B} T \gg \hbar \omega_{z}\left(k_{B}\right.$ is the Boltzmann constant), the population of the $j$ th mode is $N_{j} \simeq k_{B} T / \epsilon_{j}$ and the thermal average of the mean square fluctuations of the phase becomes

$$
\left\langle\left[\delta \hat{\phi}\left(z_{1}, z_{2}\right)\right]^{2}\right\rangle_{T}=\delta_{L}^{2}(T) f^{(1)}\left(z_{1} / L, z_{2} / L\right),
$$

where

$$
\delta_{L}^{2}(T)=\frac{32 \mu k_{B} T}{15 N_{0}\left(\hbar \omega_{z}\right)^{2}}
$$

and

$$
\begin{aligned}
f^{(1)}\left(z_{1} / L, z_{2} / L\right)= & \frac{1}{8} \sum_{j=1}^{\infty} \frac{(j+2)(2 j+3)}{j(j+1)(j+3)} \\
& \times\left[P_{j}^{(1,1)}\left(\frac{z_{1}}{L}\right)-P_{j}^{(1,1)}\left(\frac{z_{2}}{L}\right)\right]^{2},
\end{aligned}
$$

$N_{0}$ indicating the number of atoms in the condensate fraction. The first-order correlation function of the degenerate Bose gas is defined by (see, e.g., [29])

$$
g_{T}^{(1)}\left(\mathbf{r}_{1}, \mathbf{r}_{2}\right)=\frac{\left\langle\hat{\psi}^{\dagger}\left(\mathbf{r}_{1}\right) \hat{\psi}\left(\mathbf{r}_{2}\right)\right\rangle_{T}}{\left[\left\langle\hat{\psi}^{\dagger}\left(\mathbf{r}_{1}\right) \hat{\psi}\left(\mathbf{r}_{1}\right)\right\rangle_{T}\left\langle\hat{\psi}^{\dagger}\left(\mathbf{r}_{2}\right) \hat{\psi}\left(\mathbf{r}_{2}\right)\right\rangle_{T}\right]^{1 / 2}} .
$$

According to Eqs. (12) and (13), this results in

$$
g_{T}^{(1)}\left(z_{1}, z_{2}\right)=\exp \left\{-\delta_{L}^{2}(T) f^{(1)}\left(z_{1} / L, z_{2} / L\right) / 2\right\} .
$$

For $\left|z_{1}\right|,\left|z_{2}\right| \ll L$, using the asymptotic expression of the Jacobi polynomials [30] and summing over the different modes in the continuous limit, one obtains an approximated formula for the $f^{(1)}$ function valid around the center of the condensate [8]:

$$
f^{(1)}\left(z_{1} / L, z_{2} / L\right)=\left|z_{1}-z_{2}\right| / L \text {. }
$$

In that case,

$$
g_{T}^{(1)}\left(z_{1}, z_{2}\right)=\exp \left\{-\delta_{L}^{2}(T)\left|z_{1}-z_{2}\right| / 2 L\right\} .
$$

This result suggests the introduction of the phase coherence length of the condensate 


$$
L_{\phi}=\frac{L}{\delta_{L}^{2}(T)}
$$

defined as the distance at which the first-order correlation function decreases to $1 / \sqrt{e}$. The approximate formula shown in Eq. (18) can be extended to describe the behavior of the $f^{(1)}$ function far from the center of the condensate. For $\delta_{L}^{2}(T) \gg 1$, the coherence length $L_{\phi}$ is small compared to the axial size $L$ and the system is well described by means of the local density approximation $[16,17,20]$. As pointed out in Ref. [20], this limit is equivalent to the use of the approximate formula for the Jacobi polynomials with large $j$ [30]. Equation (15) can thus be written in the form

$$
f^{(1)}\left(z_{1} / L, z_{2} / L\right)=\frac{\left|z_{1}-z_{2}\right| / L}{\left[1-\left(z_{1}+z_{2}\right)^{2} /(2 L)^{2}\right]^{2}},
$$

generalizing the result obtained in Eq. (18).

We use a similar approach to calculate the two-particle correlation function of the condensate. Introducing the operator $\delta^{(2)} \hat{\phi}\left(\mathbf{r}_{1}, \mathbf{r}_{2}, \mathbf{r}_{3}, \mathbf{r}_{4}\right)=\hat{\phi}\left(\mathbf{r}_{1}\right)+\hat{\phi}\left(\mathbf{r}_{2}\right)-\hat{\phi}\left(\mathbf{r}_{3}\right)-\hat{\phi}\left(\mathbf{r}_{4}\right)$, we obtain

$$
\begin{aligned}
& \left\langle\hat{\psi}^{\dagger}\left(\mathbf{r}_{1}\right) \hat{\psi}^{\dagger}\left(\mathbf{r}_{2}\right) \hat{\psi}\left(\mathbf{r}_{3}\right) \hat{\psi}\left(\mathbf{r}_{4}\right)\right\rangle \\
& \quad=\prod_{i=1}^{4} \sqrt{n_{0}\left(\mathbf{r}_{i}\right)} \exp \left\{-\left\langle\left[\delta^{(2)} \hat{\phi}\left(\mathbf{r}_{1}, \mathbf{r}_{2}, \mathbf{r}_{3}, \mathbf{r}_{4}\right)\right]^{2}\right\rangle / 2\right\}
\end{aligned}
$$

Using Eq. (1) for the phase operator, a straightforward calculation yields

$$
\begin{aligned}
\left\langle\left[\delta^{(2)}\right.\right. & \left.\left.\hat{\phi}\left(z_{1}, z_{2}, z_{3}, z_{4}\right)\right]^{2}\right\rangle \\
= & \sum_{j=1}^{\infty} \frac{(j+2)(2 j+3) \mu}{15(j+1) \epsilon_{j} N_{0}} N_{j}\left[P_{j}^{(1,1)}\left(\frac{z_{1}}{L}\right)+P_{j}^{(1,1)}\left(\frac{z_{2}}{L}\right)\right. \\
& \left.-P_{j}^{(1,1)}\left(\frac{z_{3}}{L}\right)-P_{j}^{(1,1)}\left(\frac{z_{4}}{L}\right)\right]^{2}
\end{aligned}
$$

In the limit $k_{B} T \gg \hbar \omega_{z}$, the thermal average of Eq. (23) gives

$$
\begin{aligned}
& \left\langle\left[\delta^{(2)} \hat{\phi}\left(z_{1}, z_{2}, z_{3}, z_{4}\right)\right]^{2}\right\rangle_{T} \\
& \quad=\delta_{L}^{2}(T) f^{(2)}\left(z_{1} / L, z_{2} / L, z_{3} / L, z_{4} / L\right)
\end{aligned}
$$

where

$$
\begin{aligned}
f^{(2)}\left(z_{1} / L, z_{2} / L, z_{3} / L, z_{4} / L\right) \\
=f^{(1)}\left(z_{1} / L, z_{3} / L\right)+f^{(1)}\left(z_{2} / L, z_{4} / L\right) \\
\quad-f^{(1)}\left(z_{1} / L, z_{2} / L\right)-f^{(1)}\left(z_{3} / L, z_{4} / L\right) \\
\quad+f^{(1)}\left(z_{1} / L, z_{4} / L\right)+f^{(1)}\left(z_{2} / L, z_{3} / L\right) .
\end{aligned}
$$

Thus, the two-particle correlation function can be expressed as a product of one-particle correlation functions. Equations (18) and (21) can be used to derive simplified expressions for

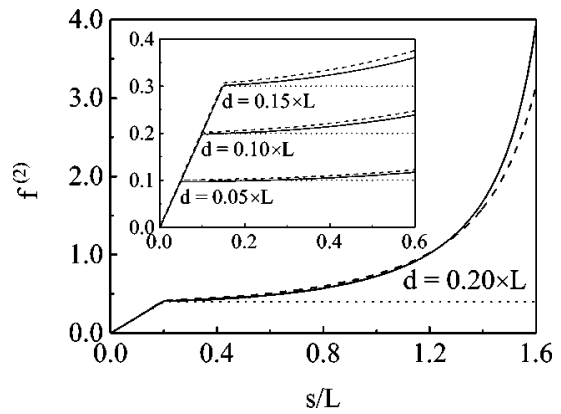

FIG. 1. $f^{(2)}\left(\bar{z}_{1} / L, \bar{z}_{2} / L, \bar{z}_{3} / L, \bar{z}_{4} / L\right)$ as a function of $s>0$. The complete expression in Eq. (25) (solid line) is compared with the approximated formulas derived from Eqs. (18) and (21), valid in the condensate center (dotted line) and in the local density approximation (dashed line). The inset shows $f^{(2)}$ for different values of $d$ $>0$.

the $f^{(2)}$ function, valid in the limit $\left|z_{i}\right| \ll L(i=1, \ldots, 4)$ and in the local density approximation. Figure 1 shows the dependence of $f^{(2)}$ calculated in

$$
\bar{z}_{1}=\frac{s+d}{2}, \quad \bar{z}_{2}=\frac{-s-d}{2}, \quad \bar{z}_{3}=\frac{s-d}{2}, \quad \bar{z}_{4}=\frac{-s+d}{2}
$$

as a function of $s>0$. The full expression of $f^{(2)}$ can be compared with the two approximated formulas, the first valid in the condensate center, the second valid in the local density approximation. The inset of Fig. 1 shows the same curves for different values of $d>0$. This choice of variables follows the particular experimental realization. In Sec. IV, we demonstrate how these curves can be characterized in a matter wave interferometry experiment by measuring density correlations in the interference pattern produced by two autocorrelated copies of a parent condensate. There, $d$ is the displacement between the two interfering condensate copies and $s$ is the separation between the positions in the interference pattern at which the particle densities are evaluated. A qualitative understanding of the behavior shown in Fig. 1 is possible if we consider that

$$
\begin{aligned}
\left\langle\left[\delta^{(2)}\right.\right. & \left.\left.\hat{\phi}\left(\bar{z}_{1}, \bar{z}_{2}, \bar{z}_{3}, \bar{z}_{4}\right)\right]^{2}\right\rangle_{T} \\
= & \left\langle\left[\delta \hat{\phi}\left(\bar{z}_{1}, \bar{z}_{3}\right)\right]^{2}\right\rangle_{T}+\left\langle\left[\delta \hat{\phi}\left(\bar{z}_{2}, \bar{z}_{4}\right)\right]^{2}\right\rangle_{T} \\
& +2\left\langle\delta \hat{\phi}\left(\bar{z}_{1}, \bar{z}_{3}\right) \delta \hat{\phi}\left(\bar{z}_{2}, \bar{z}_{4}\right)\right\rangle_{T} .
\end{aligned}
$$

The first and the second term are the thermal averages of the operator $(\delta \hat{\phi})^{2}$, calculated in $\left(\bar{z}_{1}, \bar{z}_{3}\right)$ and in $\left(\bar{z}_{2}, \bar{z}_{4}\right)$; the last term is proportional to the correlation function of $\delta \hat{\phi}$ at the same coordinates. For a fixed displacement $d$, when the examined positions are close to the condensate center $(d, s$ $\ll L$ ), the first two terms of Eq. (27) do not depend on the separation $s$. However, as $s$ rises from 0 to $d$, the third term increases from $-2\left\langle\left[\delta \hat{\phi}\left(\bar{z}_{1}, \bar{z}_{3}\right)\right]^{2}\right\rangle_{T}$ (complete anticorrelation) to its maximum value 0 , resulting in an uncorrelated phase difference for every $s \geqslant d$. In the interval $0 \leqslant s \leqslant d$, the 
$f^{(2)}$ function depends linearly on $s$ with slope 2 . The secondorder correlation function is defined as

$$
\begin{aligned}
g_{T}^{(2)} & \left(\mathbf{r}_{1}, \mathbf{r}_{2}, \mathbf{r}_{3}, \mathbf{r}_{4}\right) \\
& =\frac{\left\langle\hat{\psi}^{\dagger}\left(\mathbf{r}_{1}\right) \hat{\psi}^{\dagger}\left(\mathbf{r}_{2}\right) \hat{\psi}\left(\mathbf{r}_{3}\right) \hat{\psi}\left(\mathbf{r}_{4}\right)\right\rangle_{T}}{\left[\left\langle\hat{\psi}^{\dagger}\left(\mathbf{r}_{1}\right) \hat{\psi}\left(\mathbf{r}_{1}\right)\right\rangle_{T} \cdots\left\langle\hat{\psi}^{\dagger}\left(\mathbf{r}_{4}\right) \hat{\psi}\left(\mathbf{r}_{4}\right)\right\rangle_{T}\right]^{1 / 2}} .
\end{aligned}
$$

Substituting Eqs. (22) and (24) in Eq. (28), we obtain

$$
\begin{aligned}
& g_{T}^{(2)}\left(z_{1}, z_{2}, z_{3}, z_{4}\right) \\
& \quad=\exp \left\{-\delta_{L}^{2}(T) f^{(2)}\left(z_{1} / L, z_{2} / L, z_{3} / L, z_{4} / L\right) / 2\right\}
\end{aligned}
$$

Note that, due to the suppression of density modulations, the normalized density correlation function of the trapped condensate is constant: $g_{T}^{(2)}\left(z_{1}, z_{2}, z_{2}, z_{1}\right)=1$.

The calculation we have described for the second-order correlation function can be extended to obtain a general expression for the $\mathrm{N}$ th-order correlation function. Defining the operator

$$
\begin{aligned}
\delta^{(N)} \hat{\phi}\left(\left\{\mathbf{r}_{i}\right\}_{i=1, \ldots, 2 N}\right)= & \hat{\phi}\left(\mathbf{r}_{1}\right)+\cdots+\hat{\phi}\left(\mathbf{r}_{N}\right)-\hat{\phi}\left(\mathbf{r}_{N+1}\right) \\
& -\cdots-\hat{\phi}\left(\mathbf{r}_{2 N}\right)
\end{aligned}
$$

the $N$-particle correlation function is given by

$$
\begin{aligned}
& \left\langle\hat{\psi}^{\dagger}\left(\mathbf{r}_{1}\right) \cdots \hat{\psi}^{\dagger}\left(\mathbf{r}_{N}\right) \hat{\psi}\left(\mathbf{r}_{N+1}\right) \cdots \hat{\psi}\left(\mathbf{r}_{2 N}\right)\right\rangle \\
& \quad=\prod_{i=1}^{N} \sqrt{n_{0}\left(\mathbf{r}_{i}\right)} \exp \left\{-\left\langle\left[\delta^{(N)} \hat{\phi}\left(\left\{\mathbf{r}_{i}\right\}_{i=1, \ldots, 2 N}\right)\right]^{2}\right\rangle / 2\right\} .
\end{aligned}
$$

In general, the thermal average of the operator $\left(\delta^{(N)} \hat{\phi}\right)^{2}$ can be written in the form

$$
\left\langle\left[\delta^{(N)} \hat{\phi}\left(\left\{\mathbf{r}_{i}\right\}_{i=1, \ldots, 2 N}\right)\right]^{2}\right\rangle_{T}=\delta_{L}^{2}(T) f^{(N)}\left(\left\{z_{i} / L\right\}_{i=1, \ldots, 2 N}\right)
$$

The $f^{(N)}$ function, depending on the Jacobi polynomials $P_{j}^{(1,1)}$, can be expressed as a combination of $f^{(1)}$ functions:

$$
f^{(N)}\left(\left\{z_{i} / L\right\}_{i=1, \ldots, 2 N}\right)=\sum_{1 \leqslant l<m \leqslant 2 N} \mathcal{P}^{\{l, m\}} f^{(1)}\left(\frac{z_{l}}{L}, \frac{z_{m}}{L}\right),
$$

where the coefficient $\mathcal{P}^{\{l, m\}}$ is defined as

$$
\mathcal{P}^{\{l, m\}}= \begin{cases}+1 & \text { if } l \leqslant N<m, \\ -1 & \text { if } l, m \leqslant N \text { or } l, m>N .\end{cases}
$$

The $N$ th-order correlation function is given by

$$
\begin{aligned}
g_{T}^{(N)}( & \left.\left\{\mathbf{r}_{i}\right\}_{i=1, \ldots, 2 N}\right) \\
& =\frac{\left\langle\hat{\psi}^{\dagger}\left(\mathbf{r}_{1}\right) \cdots \hat{\psi}^{\dagger}\left(\mathbf{r}_{N}\right) \hat{\psi}\left(\mathbf{r}_{N+1}\right) \cdots \hat{\psi}\left(\mathbf{r}_{2 N}\right)\right\rangle_{T}}{\left[\left\langle\hat{\psi}^{\dagger}\left(\mathbf{r}_{1}\right) \hat{\psi}\left(\mathbf{r}_{1}\right)\right\rangle_{T} \cdots\left\langle\hat{\psi}^{\dagger}\left(\mathbf{r}_{2 N}\right) \hat{\psi}\left(\mathbf{r}_{2 N}\right)\right\rangle_{T}\right]^{1 / 2}}
\end{aligned}
$$

and, from Eqs. (31) and (32),

$$
g_{T}^{(N)}\left(\left\{z_{i}\right\}_{i=1, \ldots, 2 N}\right)=\exp \left[-\delta_{L}^{2}(T) f^{(N)}\left(\left\{z_{i} / L\right\}_{i=1, \ldots, 2 N}\right) / 2\right]
$$

This general result shows that $g_{T}^{(N)}$ is completely characterized by the parameter $\delta_{L}^{2}(T)$ which, through Eq. (20), defines $L_{\phi}$. Therefore, each correlation function contains full information about the coherence properties of the sample and can be used to measure the phase coherence length of the condensate.

\section{INTERFEROMETRIC MEASUREMENT OF THE SECOND-ORDER CORRELATION FUNCTION}

The coherence of a matter wave can be studied by using interferometric methods. However, as standard interference experiments measure the first-order correlation function of the field operator $\hat{\psi}$, they are very sensitive to phase noise introduced by the experimental apparatus. The method presented here is analogous to the original Hanbury-Brown and Twiss experiment $[23,24]$ in which the spatially resolved second-order correlation function $g^{(2)}\left(\mathbf{r}_{1}, \mathbf{r}_{2}, \mathbf{r}_{2}, \mathbf{r}_{1}\right)$ of a light source is obtained from intensity correlation measurements. In the Hanbury-Brown and Twiss interferometer the intensity of a light source is measured by two spatially displaced photodetectors. The information about the relative phase of the two light waves is therefore lost, while only correlations in their intensity fluctuations are recorded. The principle is radically different from the usual Michelson interferometer where the two waves interfere before the detection and where their relative phase plays a crucial role. We use this method to characterize the second-order correlation function of a matter wave. As discussed before, for a highly elongated BEC $g_{T}^{(2)}\left(z_{1}, z_{2}, z_{2}, z_{1}\right)=1$. This result suggests that a simple measurement of density correlations in the condensate is not sufficient to describe the coherence properties of the sample. Nevertheless, by measuring density correlations in the interference pattern generated by two spatially displaced copies of a parent BEC, it is possible to correlate the field operator $\hat{\psi}$ at four different positions and extract $g_{T}^{(2)}\left(z_{1}, z_{2}, z_{3}, z_{4}\right)$. Compared to standard interference experiments, the main advantage of this technique is the intrinsic stability of the density correlation measurement against variations of the global phase between the interfering condensates.

In this section, we show how a matter wave Bragg interferometer can be used to characterize the second-order correlation function of the condensate and to measure its phase coherence length. 


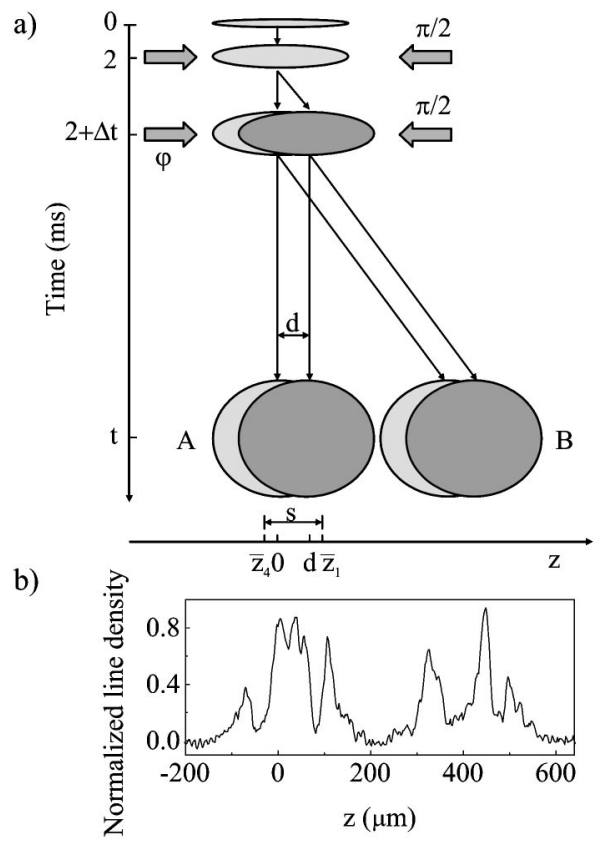

FIG. 2. (a) The matter wave Bragg interferometer: The condensate is released from the magnetic trap and, after $2 \mathrm{~ms}$ of free evolution, is interrogated by the two-pulse sequence. The first $\pi / 2$ Bragg pulse splits the parent BEC into two copies with momenta 0 and $2 \hbar k$. After a time $\Delta t$, the second $\pi / 2$ Bragg pulse splits the condensates again and allows them to interfere. The time interval $\Delta t$ defines the displacement $d$ between the two interfering BECs. (b) A typical line density profile at the output ports of the interferometer: The distance between the two autocorrelated copies $(d$ $=46 \mu \mathrm{m})$ is comparable to the phase coherence length of the parent condensate $\left(L_{\phi}=43 \mu \mathrm{m}\right)$. In the schematic of the matter wave Bragg interferometer, the distance $d$ has been exaggerated for clarity.

\section{A. Interferometric scheme}

Our interferometric sequence is shown in Fig. 2. The condensate is released from the magnetic trap and expands freely for $2 \mathrm{~ms}$. This short time of flight is important to lower the density, thus reducing $s$-wave scattering processes occurring during the Bragg diffraction of the condensate [31]. The interrogation sequence consists of two $\pi / 2$ Bragg pulses. Each pulse is composed of two counterpropagating laser beams of wave number $k$, detuned from the atomic transition. The first Bragg pulse splits the condensate in the two momentum eigenstates $|2 \hbar k\rangle$ and $|0\rangle$ along the axial direction (z). After a time $\Delta t$, a second $\pi / 2$ pulse splits the condensates again, creating two interfering copies in each momentum state. The time interval $\Delta t$ between the two pulses sets the spatial overlap $d=2 \hbar k \Delta t / m$ between the interfering BECs at the output ports of the interferometer. The relative phase of the two counterpropagating Bragg beams is externally controlled by an electro-optic modulator (EOM) and can be changed between the two pulses. This allows us to imprint an extra phase $\varphi$ which can be precisely tuned. Control of the EOM phase is crucial for our method, as described in Sec. IV B.

Using the results derived in Sec. II, the atoms detected in the output port $A$ (see Fig. 2), after a total time of flight $t$, are described by the order parameter

$$
\begin{aligned}
\psi(\mathbf{r}, d, t)= & \frac{1}{2} \sqrt{\eta\left(\mathbf{r}^{\prime}, t\right)}+\frac{1}{2} \sqrt{\eta(\mathbf{r}, t)} \exp \left\{i \left[\delta \phi\left(z, z^{\prime}, t\right)\right.\right. \\
& \left.\left.+\alpha\left(z, z^{\prime}, t\right)+\beta\left(z, z^{\prime}\right)+\gamma(d)\right]\right\},
\end{aligned}
$$

where $\mathbf{r}^{\prime}=\mathbf{r}-d \hat{\mathbf{z}}$ and $\eta(\mathbf{r}, t)$ is the time-evolved density profile normalized to the total number of atoms in the parent condensate. The relative phase between the interfering condensates contains several contributions. $\delta \phi\left(z, z^{\prime}, t\right)=\phi(z, t)$ $-\phi\left(z^{\prime}, t\right)$ describes the phase difference between $z$ and $z^{\prime}$ that evolves from the phase fluctuations in the parent condensate. The term

$$
\alpha\left(z, z^{\prime}, t\right)=\frac{m \dot{\lambda_{z}}}{2 \hbar \lambda_{z}}\left(z^{2}-z^{\prime 2}\right)
$$

represents the nonuniform spatial phase profile developed during the mean-field-driven expansion. The mean-field gradient between the interfering BECs is responsible for a force repelling the centers of mass of the two clouds. This effect is described by the phase term

$$
\beta\left(z, z^{\prime}\right)=\frac{m \delta v}{2 \hbar}\left(z+z^{\prime}\right),
$$

proportional to the relative repulsion velocity $\delta v$ between the interfering condensates [32]. After the first Bragg pulse, the relative phase of the atoms in the $|2 \hbar k\rangle$ momentum state evolves with a characteristic frequency $\delta_{\text {Bragg }}$, given by the detuning of the lasers from the resonance of the two-photon transition [33]. Therefore, the last term

$$
\gamma(d)=\delta_{\mathrm{Bragg}} \Delta t+\varphi=\delta_{\mathrm{Bragg}} \frac{m d}{2 \hbar k}+\varphi
$$

represents a global phase depending on the detuning from the Bragg transition and the externally controlled phase $\varphi$.

The density of atoms at the output port $A$ of the interferometer is given by

$$
\begin{aligned}
I(\mathbf{r}, d, t)= & \frac{1}{4} \eta(\mathbf{r}, t)+\frac{1}{4} \eta\left(\mathbf{r}^{\prime}, t\right) \\
& +\frac{1}{2} \sqrt{\eta(\mathbf{r}, t) \eta\left(\mathbf{r}^{\prime}, t\right)} \cos \left[\delta \phi\left(z, z^{\prime}, t\right)+\alpha\left(z, z^{\prime}, t\right)\right. \\
& \left.+\beta\left(z, z^{\prime}\right)+\gamma(d)\right]
\end{aligned}
$$

The presence of strong phase fluctuations alters the interference pattern generated by the two autocorrelated condensates. In fact, when $d \simeq L_{\phi}$ the phase term $\delta \phi$ can be comparable to $\pi$, modifying drastically and in an unpredictable way the position and the spacings of the interference fringes.

\section{B. Method}

Starting from Eq. (41), we want to calculate the density correlation function of the interference pattern for an ensemble of identically prepared condensates at a given tem- 
perature $T$, averaged over all the global phase values $\varphi$. This averaging process is indicated by the symbol $\langle\cdots\rangle_{T, \varphi}$. It is therefore important that the phase delay $\varphi$ induced by the EOM is uniformly changed between 0 and $2 \pi$. In Sec. II, we showed that, for typical times of flight (tens of milliseconds), the evolution of the fluctuating phase of the condensate is basically frozen. This allows us to neglect the time dependence of $\delta \phi\left(z, z^{\prime}, t\right)$. We also neglect the contribution of density modulations induced by the initial phase pattern on the Thomas-Fermi profile of the condensate. The validity of this approximation is verified below. Under these assumptions, we calculate the normalized density correlation function

$$
\gamma^{(2)}\left(\mathbf{r}_{1}, \mathbf{r}_{2}, d, t\right)=\frac{\left\langle\left(I_{1}-\left\langle I_{1}\right\rangle_{T, \varphi}\right)\left(I_{2}-\left\langle I_{2}\right\rangle_{T, \varphi}\right)\right\rangle_{T, \varphi}}{\sqrt{\left\langle\left(I_{1}-\left\langle I_{1}\right\rangle_{T, \varphi}\right)^{2}\right\rangle_{T, \varphi}\left\langle\left(I_{2}-\left\langle I_{2}\right\rangle_{T, \varphi}\right)^{2}\right\rangle_{T, \varphi}}},
$$

where $I_{1,2}=I\left(\mathbf{r}_{1,2}, d, t\right)$. After a lengthy but straightforward calculation, the averaging process gives

$$
\begin{aligned}
\gamma^{(2)}\left(z_{1}, z_{2}, d, t\right)= & \cos \left[\frac{m}{\hbar}\left(\frac{\dot{\lambda_{z}}}{\lambda_{z}} d+\delta v\right)\left(z_{1}-z_{2}\right)\right] \\
& \times \exp \left[-\delta_{L}^{2}(T) f^{(2)}\left(z_{1} / L,\left(z_{2}-d\right) / L\right.\right. \\
& \left.\left.\left(z_{1}-d\right) / L, z_{2} / L\right) / 2\right] .
\end{aligned}
$$

$\gamma^{(2)}\left(z_{1}, z_{2}, d, t\right)$ results from the product of two different terms: the first is a periodic function, whose argument is the contribution of the mean-field energy to the phase profile (ballistic expansion and relative repulsion between the interfering condensates); the second is an exponential term which corresponds to the $g_{T}^{(2)}$ function of the parent phase fluctuating condensate [see Eq. (29)]. The decay constant of this function is given by the phase coherence length of the condensate [see Eq. (20)].

From the experimental point of view, the averaging process described above is equivalent to the following procedure. The radially integrated density profile $I=I(z, d, t)$ at the output port $A$ of the interferometer is measured for different values of the global phase, uniformly distributed in the range $0 \leqslant \varphi<2 \pi$; then, the average value $\langle I\rangle_{T, \varphi}$ is calculated and used to determine $I-\langle I\rangle_{T, \varphi}$ for each experimental realization. These profiles, averaged according to Eq. (42), give a measurement of $\gamma^{(2)}\left(z_{1}, z_{2}, d, t\right)$. For simplicity, we choose symmetric positions around the center $(z=d / 2)$ of the interference pattern and evaluate density correlations as a function of the separation $s=z_{2}-z_{1}$. According to Eq. (26), this particular choice corresponds to $z_{1}=(s+d) / 2=\bar{z}_{1}$ and $z_{2}$ $=(-s+d) / 2=\bar{z}_{4}$ (see also Fig. 2). Substituting in Eq. (43) and taking into account Eqs. (26) and (29), we obtain

$$
\begin{aligned}
\gamma^{(2)}(s, d, t)= & \cos \left[\frac{m}{\hbar}\left(\frac{\dot{\lambda_{z}}}{\lambda_{z}} d+\delta v\right) s\right] \\
& \times \exp \left[-\delta_{L}^{2}(T) f^{(2)}\left(\bar{z}_{1} / L, \bar{z}_{2} / L, \bar{z}_{3} / L, \bar{z}_{4} / L\right) / 2\right] \\
= & \cos \left[\frac{m}{\hbar}\left(\frac{\dot{\lambda_{z}}}{\lambda_{z}} d+\delta v\right) s\right] g_{T}^{(2)}\left(\bar{z}_{1}, \bar{z}_{2}, \bar{z}_{3}, \bar{z}_{4}\right) .
\end{aligned}
$$

The method described here allows us to characterize the dependence of the correlation function on the separation $s$ for any fixed displacement $d$ between the interfering condensates.

\section{Experimental results and numerical simulations}

We perform the experiment with ${ }^{87} \mathrm{Rb}$ condensates in the $F=1, m_{F}=-1$ state. The atoms are confined in a highly elongated magnetic trap with cylindrical symmetry, the long axis lying in the horizontal plane. The confining potential has an axial frequency $\omega_{z}=2 \pi \times 3.4 \mathrm{~Hz}$ and a radial frequency $\omega_{\rho}$ which is varied between $2 \pi \times 300 \mathrm{~Hz}$ and $2 \pi$ $\times 380 \mathrm{~Hz}$. Further details on the experimental apparatus can be found in [18]. After the BEC formation, we let the system thermalize in the magnetic trap for typically $4 \mathrm{~s}$ in the presence of radio frequency shielding [34]. That time is important to reach an equilibrium condition in which any quadrupole oscillation has been damped down. As shown in Fig. 2, our matter wave interferometer consists of two $\pi / 2$ Bragg diffraction pulses. Each of them is composed of two counterpropagating laser beams, detuned by about $3 \mathrm{GHz}$ from the atomic transition. This detuning suppresses spontaneous scattering of photons during the interrogation time. The Bragg pulse duration of $100 \mu \mathrm{s}$ is sufficiently short not to resolve the momentum distribution of the atoms in the condensate and long enough to avoid higher-order Bragg diffraction processes. A fixed frequency difference is set between the two counterpropagating beams to match the Bragg condition. The condensate is released from the magnetic trap and after $2 \mathrm{~ms}$ of time of flight is probed by the two-pulse sequence of the interferometer. The atomic cloud is detected after the ballistic expansion by resonant absorption imaging.

Figure 2(b) shows a typical line density profile of an interference pattern in which the distance between the two autocorrelated copies $(d=46 \mu \mathrm{m})$ is comparable to the phase coherence length of the parent condensate $\left(L_{\phi}=43 \mu \mathrm{m}\right)$. Because of the stochastic nature of phase fluctuations, the fringe spacing is not regular and differs in each experimental realization. This demonstrates that the phase of the condensate can significantly change on distances comparable with the phase coherence length of the sample. Even if each single image shows high contrast, the interference pattern is completely washed out when we average a significant number of realizations.

The results of standard interference experiments are related to the correlations of the wave function and therefore are very sensitive to phase instabilities. Figure 3 shows the interference signal obtained by measuring the number of atoms in an interval of width $0.2 L$ around the center of the interference pattern $(z=d / 2)$ at the output port $A$, as a function of the global phase $\varphi$ controlled by the EOM. This signal is normalized to the corresponding number of atoms in the parent condensate. The two plots correspond to different displacements $d$ between the interfering condensates. A small displacement is related to a short time interval between the two interrogation Bragg pulses. In that case, the contribution of phase fluctuations and the effect of technical phase noise introduced by the experimental apparatus are both negligible. 


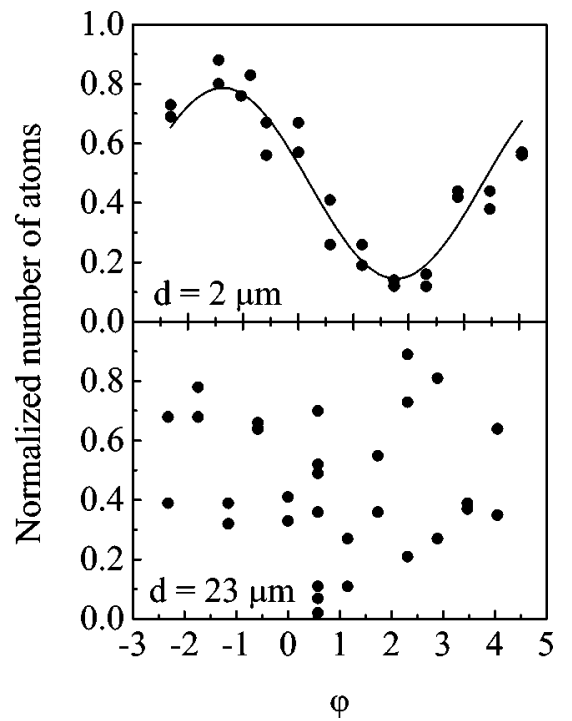

FIG. 3. The number of atoms measured in the interval $d / 2$ $-0.1 L<z<d / 2+0.1 L$, around the center of the interference pattern detected at the output port $A$, is plotted as a function of the phase $\varphi$ controlled by the electro-optic modulator. The signal is normalized to the corresponding number of atoms in the parent condensate. The two sets of data correspond to different displacements $d$ between the overlapping condensates. The solid line is obtained by fitting the experimental data with a sinusoidal function. The measurements refer to condensates with about $3 \times 10^{5}$ atoms, a typical axial size of $L=180 \mu \mathrm{m}$, and a temperature $T=170 \mathrm{nK}$.

Therefore, according to Eq. (41), when $d \ll L_{\phi}$ and $\Delta t$ is small compared to the characteristic time stability of our Bragg pulses, the normalized signal oscillates sinusoidally with high contrast. For $d$ approaching $L_{\phi}$, the random phase introduced by phase fluctuations washes out the oscillation. On average, when external disturbances can be neglected, the contrast of the oscillations is directly related to the first-order correlation function $g_{T}^{(1)}$ at a given displacement $d$. However, as $d$ increases, the external disturbances [35] also increase and produce a random phase noise which destroys the oscillating behavior and hides the effect of phase fluctuations on the detected signal.

This problem can be solved by using the method described in Sec. IV B. The measurement of intensity correlations, in combination with the subsequent averaging process, has the major advantage of being insensitive to technical phase noise introduced by the experimental apparatus. Figure 4 shows the correlation function $\gamma^{(2)}(s, d, t)$ extracted from a set of 29 line density profiles corresponding to $5.0 \times 10^{5}$ condensed atoms at a temperature $T=216 \mathrm{nK}$, detected after a total time of flight $t=37 \mathrm{~ms}$. The displacement between the interfering BECs is $d=35 \mu \mathrm{m}$. The experimental data are compared with a numerical simulation which produces random phase patterns according to the experimental conditions and uses Eq. (37) to describe the evolution of the order parameter. The numerically calculated points shown in Fig. 4 are obtained by following the same averaging procedure we have applied to the experimental data. This kind of analysis includes the time dependence of the fluctuating phase and of density modulations induced by the initial phase pattern. The

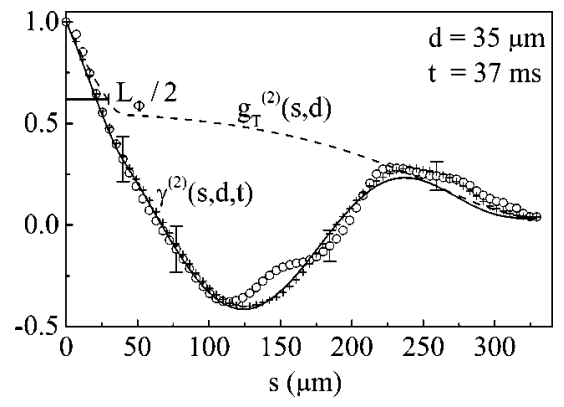

FIG. 4. Open circles: Correlation function $\gamma^{2}(s, d, t)$ extracted from a set of 29 line density profiles. The data correspond to samples with $5.0 \times 10^{5}$ condensed atoms at a temperature $T$ $=216 \mathrm{nK}$, detected after a total time of flight $t=37 \mathrm{~ms}$. The displacement between the interfering BECs is $d=35 \mu \mathrm{m}$. The bars on the experimental points represent the statistical errors. Crosses: $\mathrm{Nu}-$ merical simulation that takes into account the time dependence of the fluctuating phase and of density modulations, modeled on the experimental parameters. Solid line: Fit to the experimental data using the model function of Eq. (45). Dashed line: Second-order correlation function $g_{T}^{(2)}(s, d)=g_{T}^{(2)}\left(\bar{z}_{1}, \bar{z}_{2}, \bar{z}_{3}, \bar{z}_{4}\right)$ extracted from the fit to the experimental data. The phase coherence length of the sample is graphically indicated on the plot.

solid line is the result of a fit to the experimental data. According to Eq. (44), the model function

$$
\cos (a s) \exp \left[-b f^{(2)}\left(\bar{z}_{1} / L, \bar{z}_{2} / L, \bar{z}_{3} / L, \bar{z}_{4} / L\right) / 2\right]
$$

contains only two free parameters. The curves clearly show the damped oscillating behavior. The oscillation frequency strictly defines the parameter $a$, while the damping coefficient gives a measurement of $\delta_{L}^{2}(T)$. From the fitting function it is possible to extract the spatial dependence of the second-order correlation function $g_{T}^{(2)}(s, d)$ $=g_{T}^{(2)}\left(\bar{z}_{1}, \bar{z}_{2}, \bar{z}_{3}, \bar{z}_{4}\right)$ [see Eq. (44)]. The fit on the experimental data gives a phase coherence length $L_{\varphi}^{\text {expt }}=57 \pm 10 \mu \mathrm{m}$, compatible with the expected value $L_{\varphi}^{\text {theor }}=58 \pm 2 \mu \mathrm{m}$. The good agreement between the experimental data, the numerical simulation, and the model function of Eq. (45) demonstrates that the free evolution of density modulations and of the fluctuating phase pattern does not influence the measurement of the second-order correlation function. This result justifies the use of Eq. (44) to model the experimental data and to extract the coherence properties of the condensate.

In Fig. 5, we show a direct comparison between the measured phase coherence lengths in the center of the BEC and the theoretical values calculated according to Eq. (20) by using the measured numbers of atoms, temperatures, and trapping frequencies. The bars indicate the statistical errors both on the measured values and on the theoretical predictions. The dotted line with slope 1 highlights the good quantitative agreement between experiments and theory.

\section{CONCLUSION}

In this paper we have studied the coherence properties of phase fluctuating Bose-Einstein condensates. In highly elongated BECs the thermal excitation of quasiparticle modes 


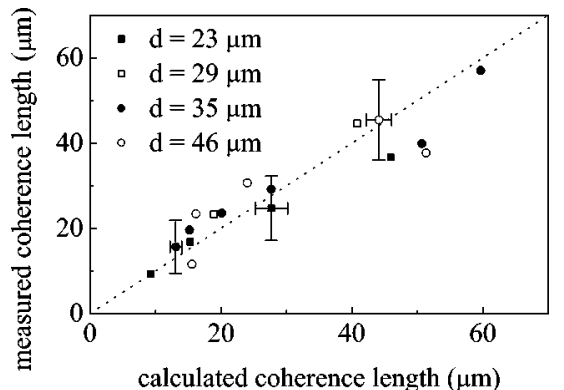

FIG. 5. Direct comparison between the measured phase coherence lengths and the theoretical values, calculated according to Eq. (20) by using the measured numbers of atoms, temperatures, and trapping frequencies. The dotted line with slope 1 is used to compare experiment and theory. The bars on the plotted points indicate statistical errors. The relative systematic uncertainties on calculated and measured phase coherence lengths are $26 \%$ and $15 \%$, respectively. This figure has previously been shown in [22].

can significantly reduce the coherence length of the system. Starting from the results of Petrov et al. [8], we have derived a general formula for the $\mathrm{N}$-particle correlation function. The second-order correlation function has been studied in detail and its limits both around the center of the condensate and in the local density approximation have been analyzed. In particular, we have discussed a method to directly characterize the second-order correlation properties of the system. An analytic theory that describes the free evolution of the condensate phase has been developed to closely model the measurement process. Using a Bragg diffraction interferometer, we have measured the density correlations of the interference pattern generated by two spatially displaced copies of a parent BEC. This kind of measurement allows a correlation of the field operator $\hat{\psi}$ of the parent condensate in four different $z$ positions. The averaging process directly gives the secondorder correlation function. The experiment confirms our theoretical predictions and demonstrates a method to measure the phase coherence length of the condensate. Compared to the usual interference experiments, this technique has the advantage of being insensitive to the global phase noise introduced by the experimental apparatus. The method presented here is in direct analogy to the original HanburyBrown and Twiss experiment and demonstrates the possibility of using density correlation measurements to study the coherence properties of Bose-Einstein condensates.

\section{ACKNOWLEDGMENTS}

We gratefully thank DFG for support in the Sonderforschungsbereich 407 as well as the European Union for support in the RTN network "Preparation and application of quantum-degenerate cold atomic/molecular gases," Contract No. HPRN-CT-2000-00125. L.S. thanks the Alexander von Humboldt Foundation and the ZIP program of the German Government for support.
[1] F. Dalfovo, S. Giorgini, L.P. Pitaevskii, and S. Stringari, Rev. Mod. Phys. 71, 463 (1999).

[2] M.R. Andrews, C.G. Townsend, H.-J. Miesner, D.S. Durfee, D.M. Kurn, and W. Ketterle, Science 275, 637 (1997).

[3] E.W. Hagley, L. Deng, M. Kozuma, M. Trippenbach, Y.B. Band, M. Edwards, M. Doery, P.S. Julienne, K. Helmerson, S.L. Rolston, and W.D. Phillips, Phys. Rev. Lett. 83, 3112 (1999).

[4] J. Stenger, S. Inouye, A.P. Chikkatur, D.M. Stamper-Kurn, D.E. Pritchard, and W. Ketterle, Phys. Rev. Lett. 82, 4569 (1999).

[5] D.S. Petrov, G.V. Shlyapnikov, and J.T.M. Walraven, Phys. Rev. Lett. 85, 3745 (2000).

[6] D.S. Petrov, M. Holtzmann, and G.V. Shlyapnikov, Phys. Rev. Lett. 84, 2551 (2000).

[7] Yu. Kagan, V.A. Kashurnikov, A.V. Krasavin, N.V. Prokof'ev, and B.V. Svistunov, Phys. Rev. A 61, 043608 (2000).

[8] D.S. Petrov, G.V. Shlyapnikov, and J.T.M. Walraven, Phys. Rev. Lett. 87, 050404 (2001).

[9] T.-L. Ho and M. Ma, J. Low Temp. Phys. 115, 61 (1999).

[10] J.W. Kane and L.P. Kadanoff, Phys. Rev. 155, 80 (1967).

[11] D.M. Gangardt and G.V. Shlyapnikov, Phys. Rev. Lett. 90, 010401 (2003).

[12] J.O. Andersen, U. Al Khawaja, and H.T.C. Stoof, Phys. Rev. Lett. 88, 070407 (2002).

[13] U. Al Khawaja, N.P. Proukakis, J.O. Andersen, M.W.J. Romans, and H.T.C. Stoof, e-print cond-mat/0303483.
[14] D.L. Luxat and A. Griffin, Phys. Rev. A 67, 043603 (2003).

[15] C. Mora and Y. Castin, Phys. Rev. A 67, 053615 (2003).

[16] S. Dettmer, D. Hellweg, P. Ryytty, J.J. Arlt, W. Ertmer, K. Sengstock, D.S. Petrov, G.V. Shlyapnikov, H. Kreutzmann, L. Santos, and M. Lewenstein, Phys. Rev. Lett. 87, 160406 (2001).

[17] D. Hellweg, S. Dettmer, P. Ryytty, J.J. Arlt, W. Ertmer, K. Sengstock, D.S. Petrov, G.V. Shlyapnikov, H. Kreutzmann, L. Santos, and M. Lewenstein, Appl. Phys. B: Lasers Opt. 73, 781 (2001).

[18] H. Kreutzmann, A. Sanpera, L. Santos, M. Lewenstein, D. Hellweg, L. Cacciapuoti, M. Kottke, T. Schulte, K. Sengstock, J.J. Arlt, and W. Ertmer, Appl. Phys. B: Lasers Opt. 76, 165 (2003).

[19] S. Richard, F. Gerbier, J.H. Thywissen, M. Hugbart, P. Bouyer, and A. Aspect, Phys. Rev. Lett. 91, 010405 (2003).

[20] F. Gerbier, J.H. Thywissen, S. Richard, M. Hugbart, P. Bouyer, and A. Aspect, Phys. Rev. A 67, 051602 (2003).

[21] I. Shvarchuck, C. Buggle, D.S. Petrov, K. Dieckmann, M. Zielonkowski, M. Kemmann, T.G. Tiecke, W. von Klitzing, G.V. Shlyapnikov, and J.T.M. Walraven, Phys. Rev. Lett. 89, 270404 (2002).

[22] D. Hellweg, L. Cacciapuoti, M. Kottke, T. Schulte, K. Sengstock, W. Ertmer, and J.J. Arlt, Phys. Rev. Lett. 91, 010406 (2003).

[23] R. Hanbury-Brown and R.Q. Twiss, Nature (London) 177, 27 (1956). 
[24] R. Hanbury-Brown and R.Q. Twiss, Nature (London) 178, 1046 (1956).

[25] S.I. Shevchenko, Sov. J. Low Temp. Phys. 18, 223 (1992).

[26] S. Stringari, Phys. Rev. A 58, 2385 (1998).

[27] Yu. Kagan, E.L. Surkov, and G.V. Shlyapnikov, Phys. Rev. A 54, R1753 (1996).

[28] Y. Castin and R. Dum, Phys. Rev. Lett. 77, 5315 (1996).

[29] M. Scully and M. Zubairy, Quantum Optics (Cambridge University Press, Cambridge, U.K., 1997).

[30] I.S. Gradshteyn and I.M. Ryzhik, Table of Integrals, Series and Products (Academic Press, New York, 1965).

[31] A.P. Chikkatur, A. Görlitz, D.M. Stamper-Kurn, S. Inouye, S. Gupta, and W. Ketterle, Phys. Rev. Lett. 85, 483 (2000).

[32] J.E. Simsarian, J. Denschlag, M. Edwards, C.W. Charles, L.
Deng, E.W. Hagley, K. Helmerson, S.L. Roltson, and W.D. Phillips, Phys. Rev. Lett. 85, 2040 (2000).

[33] D.S. Weiss, B.C. Young, and S. Chu, Appl. Phys. B: Lasers Opt. 59, 217 (1994).

[34] We measure the typical thermalization times of our condensates by performing experiments of BEC growth. After times of a few hundreds of milliseconds the system reaches an equilibrium condition.

[35] For example, a change in the release velocity of 5.5 $\times 10^{-3} v_{\text {rec }}$, where $v_{\text {rec }}=\hbar \mathrm{k} / \mathrm{m}=5.9 \mu \mathrm{m} / \mathrm{ms}$ is the recoil velocity on the $D_{2}$ line, leads to a phase change of $\pi / 2$ during a time $\Delta t=3 \mathrm{~ms}$ between the Bragg pulses. This value approximately corresponds to the stability of the release velocity in our experimental apparatus. 